Después del Consenso de Washington

Relanzando el crecimiento y

las reformas en América Latina

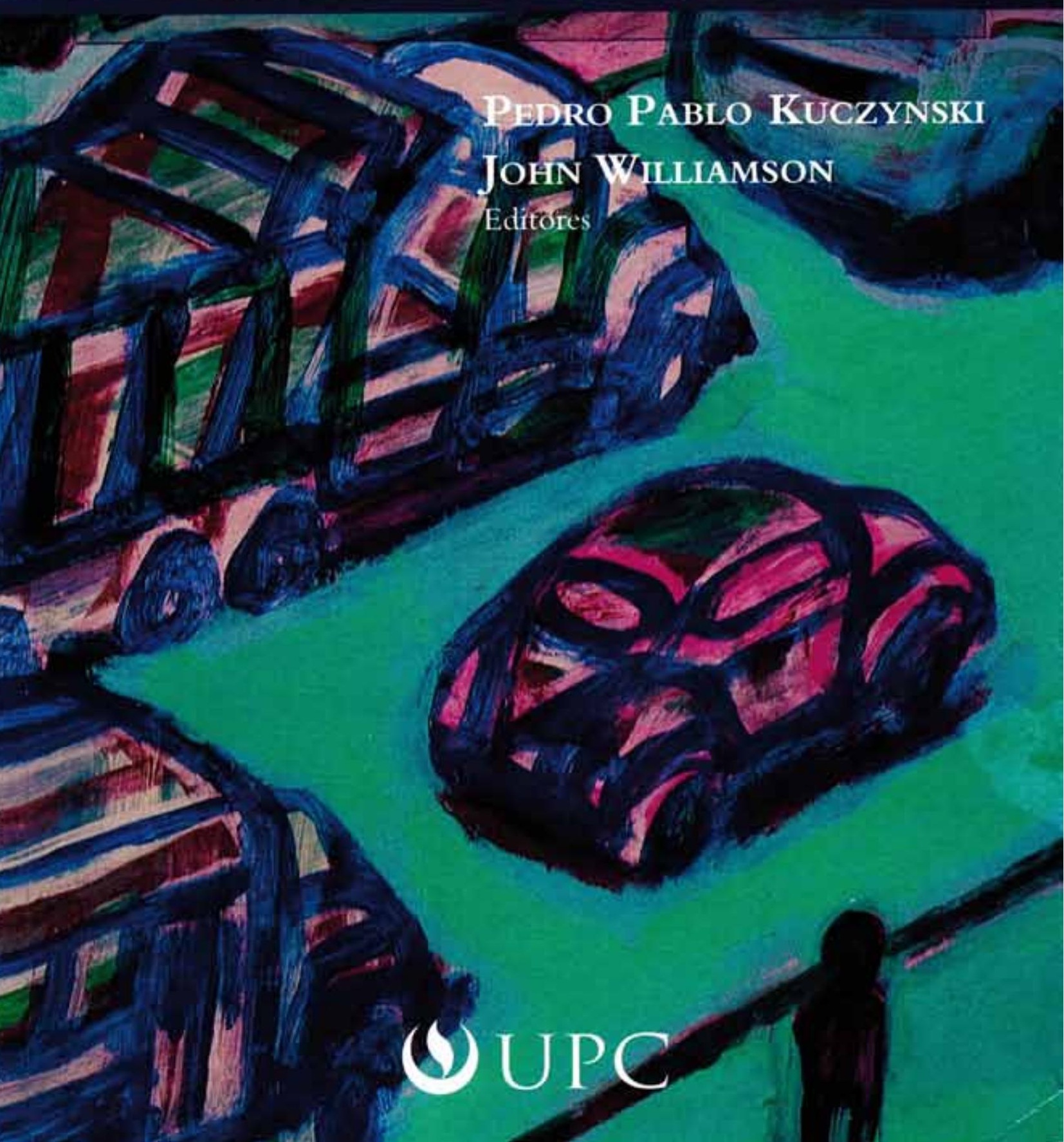




\section{Después del}

\section{Consenso de Washington}

Relanzando el crecimiento y

las reformas en América Latina

\section{Pedro Pablo Kuczynski \\ JOHN Williamson \\ Editores}

Lima, diciembre de 2003 
Título original: After the Washington Consensus

Restarting Growth and Reform in Latin America

C 2003, The Institute for International Economics

(C) De la traducción: Universidad Peruana de Ciencias Aplicadas (UPC) Publicada por acuerdo con The Institute for International Economics Diciembre 2003

Impreso en el Perú

Cubierta: Piero Quijano

Traductores: Langrow English to Grow

Corrección de estilo: Alejandra Núñez

Diseño de cubierta y diagramación: Taller Cuatro

\section{Universidad Peruana de Ciencias Aplicadas}

\section{Centro de Información}

Kuczynski, Pedro Pablo y Williamson, John (Editores)

Después del Consenso de Washington

Relanzando el crecimiento y las reformas en América Latina

Universidad Peruana de Ciencias Aplicadas (UPC), 2003.

ISBN 9972-676-72-2

Hecho el depósito legal en la Biblioteca Nacional del Perú

1501052003-6730

338.98 / KUCZ

DESARROLLO ECONÓMICO/CRECIMIENTO

ECONÓMICO/ESTADO/POLÍTICA MONETARIA/POLÍTICA FISCAL/POLITICA SOCIAL/SISTEMA FINANCIERO/ MERCADO LABORAL/AMÉRICA LATINA 
La Universidad Peruana de Ciencias Aplicadas (UPC) agradece a la Embajada de los Estados Unidos de América por el valioso apoyo que hizo posible la presente edición. 


\section{INSTITUTO DE ECONOMÍA INTERNACIONAL WASHINGTON DC MARzo 2003}

John Williamson, profesor asociado senior del Instituto de Economía Internacional desde 1981, fue director del Panel de Alto Nivel de la ONU en Finanzas para el Desarrollo (Informe Zedillo) en el 2001. Se desempeñó como economista en jefe para el Sur de Asia en el Banco Mundial durante 1996-1999; profesor de Economía en la Pontificia Universidade Católica do Rio de Janeiro (1978-1981), Universidad de Warwick (1970-1977), en el Massachussets Institute of Technology $(1967,1980)$, en la Universidad de York (1963-1968) y en la Universidad de Princeton (1962-1963); asesor ante el Fondo Monetario Internacional (1972-1974) y asesor económico del UK Treasury (1968-1970). Ha sido autor, coautor, editor o coeditor de numerosos estudios en monetarismo internacional y temas de deuda de los países en desarrollo, incluidos Sobrevaluación del dólar y la economía mundial (2003), Confianza en el alivio de la deuda; Del oro del FMI a una nueva arquitectura de asistencia (2002) y Regímenes de tipo de cambio para mercados emergentes: Reviviendo la opción intermedia (2000).

Pedro Pablo Kuczynski fue ministro de Economía y Finanzas del Perú en 2001-2002 y ministro de Energía y Minas en 1980-1982. Ha sido presidente y CEO del Latin America Enterprise Fund LP desde sus inicios en 1994. De 1992 a 1994, fue vicedirector de CAP S.A., una compañía chilena de productos de acero y forestales. De 1982 a 1992 fue director del First Boston International y director administrativo del First Boston Corporation. También ha sido socio en Kuhn, Loeb \& Company International (1973-1975), subdirector del Banco Central de Reserva del Perú (1967-1969) y presidente y CEO de Halco Mining (1977-1980). Inició su carrera en el Banco Mundial en 1961 y ocupó altos puestos en dicha institución y en la Corporación Financiera Internacional. Es autor de varios libros sobre la economía de América Latina. 


\section{ÍNDICE}

Presentación de la Edición en Español

Prefacio

Agradecimientos

Visión General:

Una Agenda para Relanzar el Crecimiento y las Reformas John Williamson

1. Explicando el Contexto

Pedro Pablo Kuczynski

2. Reformando el Estado

Pedro Pablo Kuczynski

3. Autogestión, no Soluciones Temporales:

Pobreza, Equidad y Política Social

Nancy Birdsall y Miguel Székely

4. Una Agenda de Política Fiscal

Daniel Artana, Ricardo López Murphy y Fernando Navajas

5. El Sistema Financiero

Pedro Pablo Kuczynski

6. Política Monetaria y Tipos de Cambio:

Principios Normativos para un Régimen Sostenible

Liliana Rojas-Suarez 


\section{Presentación de la Edición en Español}

El debate sobre el Consenso de Washington comenzó recién a fines de los años noventa, cuando, junto con los demás países emergentes, América Latina inició un largo proceso recesivo.

Hacia fines de los años ochenta, era claro que la gran mayoría de países de América Latina había fracasado en su modelo de desarrollo. El crecimiento basado en la sustitución de importaciones, impulsada desde la Comisión Económica para América Latina (CEPAL), y la hostilidad hacia el capital extranjero originada en la teoría de dependencia, manifestaron toda su inoperancia cuando ocurrió la crisis de la deuda externa. El escenario era evidentemente caótico y su relación con la deficiente gestión de los Estados saltaba a los ojos: altos déficit públicos, mercados financieros reprimidos, mercados cambiarios artificialmente controlados, mercados de bienes cerrados a la competencia mundial, restricciones a la inversión extranjera, empresas estatales ineficientes, rígidas legislaciones laborales y derechos de propiedad mal definidos. La relación entre este modelo de gestión económica y la recesión generalizada, la alta inflación y los problemas de balanza de pagos, no eran ya motivo de debate. Era evidente para toda mente sensata.

Cuando a fines de dicha década — la "década perdida" para América Latina - Bela Balassa convoca a un grupo de economistas, entre los que estaban Pedro Pablo Kuczynski y John Williamson, para promover un renovado crecimiento en América Latina, ya era entonces evidente el contraste entre el estancamiento latinoamericano y el éxito de países como los del Este asiático que habían orientado sus esfuerzos a la exportación. El Consenso de Washington no era, pues, solo el de las multilaterales basadas en Washington. Era también consenso del grueso de sectores dirigentes de América Latina. Recogía el sentido común sobre la necesidad de revertir el modelo cerrado y estatista que había fracasado, emprendiendo un conjunto de reformas de ordenamiento macroeconómico y de liberalización de los mercados. 
Durante los años inmediatamente posteriores a 1990, cuando John Williamson acuñó el término, había, pues, consenso. Pocos cuestionaban las reformas en favor del libre mercado emprendidas por la mayoría de países de la región y menos aún los resultados en términos de equilibrios macroeconómicos y crecimiento.

Lo que siguió a las recomendaciones del Consenso fue la aplicación desigual de dichas medidas en cada uno de los diferentes países de América Latina. Los que las aplicaron con mayor convicción y de manera más institucionalizada son los que mejor desempeño han tenido. En primer lugar, Chile, que había empezado su reforma antes que los demás países, y cuyo gran logro durante los noventa fue sin duda alcanzar un consenso político alrededor del modelo de economía de mercado, en el marco de un cada vez más sólido sistema democrático. En el caso de México, la clave fue su ventajosa integración al mercado norteamericano, demostrando una vez más que con el libre comercio el país pequeño tiene más que ganar que el grande.

Progresivamente, sin embargo, el péndulo ideológico nos ha llevado a la reaparición de la demagogia populista en la región y, lamentablemente, el término "Consenso de Washington" ha sido perversamente utilizado para atacar los cimientos mismos de la economía de mercado. En estas circunstancias, este libro - cuya edición en español presentamos en esta ocasión- es particularmente importante para el análisis del crecimiento económico y de las reformas en América Latina, ya que responde de manera alturada y técnica a las críticas ideológicas populistas. Pero, sobre todo, porque pone en agenda la necesidad de ir más allá de las recomendaciones del Consenso, no para negarlo ni mediatizarlo sino para complementarlo con reformas adicionales teniendo siempre como horizonte la exigencia de impulsar un mayor crecimiento en la región.

El caso más polémico y utilizado para criticar al Consenso ha sido el de la crisis de Argentina. Lo paradójico es que, como se muestra en este libro, los dos grandes problemas que la desencadenaron fueron en realidad las violaciones al espíritu del Consenso, sin duda avaladas en su momento erradamente por el Fondo Monetario Internacional. La primera fue la camisa de fuerza del ancla cambiaria. No dejar libre el mercado cambiario - como se hizo con éxito en Perú- impidió que en dicho país las devaluaciones sirvieran como mecanismo de ajuste. No obstante, fue la crisis en el manejo de las finanzas públicas la que en última instancia lanzó el "mecanismo de crisis" que desembocó en el cataclismo económico de fines de 2001 .

Los otrora defensores del fracasado modelo de sustitución de importaciones y de la activa intervención del Estado en la economía han resucitado identificando al Consenso de Washington con las reformas 
prioritario. Los artículos que forman parte del presente volumen, todos de autores de primer nivel que conocen de cerca las economías latinoamericanas, precisan la agenda sobre la base de la cual debemos trabajar.

Para la Universidad Peruana de Ciencias Aplicadas (UPC) es particularmente grato presentar esta edición en español, de manera coincidente con la Primera Convención Anual de Economía organizada por la Facultad de Economía de esta casa de estudios. Durante esta Convención, titulada Instituciones, Competitividad y Crecimiento, John Williamson y Pedro Pablo Kuczynski se encuentran con Douglass C. North, Premio Nobel de Economía 1993 y principal exponente de la Nueva Economía Institucional, cuyo aporte a la teoría económica debe ser utilizado para seguir enriqueciendo la agenda de reformas para el crecimiento.

De esta forma, la Universidad Peruana de Ciencias Aplicadas (UPC) y su Facultad de Economía se proponen contribuir con la difusión de estudios y argumentos que oxigenen el debate académico y cumplir una tarea de docencia económica y ciudadana. Porque nuestra Universidad entiende que ése es el mejor modo de seguir impulsando políticas y reformas que redunden en favor del desarrollo de América Latina.

Luis Bustamante Belaunde

Rector

Daniel Córdova Cayo

Decano de la Facultad de Economía

Diciembre de 2003 


\section{PREFACIO}

América Latina ha sido foco de investigación para el Instituto de Economía Internacional durante toda su vida académica. Nuestros numerosos estudios sobre la crisis de la deuda de los años ochenta se centraron desde luego en esa región. Ejemplos de nuestro trabajo incluyen el análisis de John Williamson de las Perspectivas de ajuste en Argentina, Brasil $\gamma$ México: Respondiendo a la crisis de la deuda (1983), Inflación e indexación: Argentina, Brasil e Israel (1985), y los altamente influyentes Deuda internacional y la estabilidad de la economía mundial (1983) y Deuda internacional: Riesgo sistémico $y$ respuesta de política (1984) de William $\mathrm{R}$. Cline.

A mediados de la década del ochenta, empezamos a mirar "más allá de la crisis de la deuda" en un esfuerzo por trazar un camino para restablecer la prosperidad sostenida en la región. Como parte central de ese esfuerzo reunimos un equipo de importantes economistas latinoamericanos (Gerardo Bueno de México, Pedro Pablo Kuczynski de Perú y Mario Henrique Simonsen de Brasil) para trabajar con nuestro propio investigador senior Bela Balassa y producir Hacia un crecimiento renovado en América Latina, el cual proponía un plan para ese fin. Evaluamos el humor cambiante de la región en una conferencia realizada en 1989 , cuyos pormenores fueron publicados en El progreso de la reforma de política en América Latina en 1990, donde el editor John Williamson acuñó la frase "el Consenso de Washington".

Este nuevo estudio da una mirada fresca al desarrollo durante la década siguiente en un esfuerzo por entender por qué los resultados de la reforma, a pesar de ser claramente positivos en muchos aspectos, son aún tan decepcionantes. Evalúa los éxitos y fracasos de las reformas de política actuales y desarrolla una agenda política sintonizada con la situación. Lo titulamos deliberadamente Después del Consenso de Washington para enfatizar nuestra creencia de que ya es tiempo de que el mundo deje atrás los tendenciosos debates ideológicos en los cuales el Consenso de Washington es caricaturizado como un manifiesto neoliberal, y discuta seriamente acerca 
de la nueva ola de reformas que la región necesita para reiniciar su crecimiento y ser más equitativa de lo que ha sido en el pasado.

Este volumen ha sido producido en gran parte por economistas de América Latina, cada uno de los cuales se ocupa de su área de especialidad. Doce de los 15 autores son latinoamericanos, incluyendo dos ex ministros de Economía (Pedro Pablo Kuczynski de Perú y Ricardo López Murphy de Argentina). De hecho, la idea del proyecto surgió de un latinoamericano, el doctor Kuczynski (antes de su reingreso al gobierno de su país en el 2001, lo cual retrasó la culminación de este volumen), quien fue coautor de nuestro estudio anterior en 1986 y creía, con razón, que había llegado el momento de hacer una completa reevaluación del estado de América Latina y de lo que se necesita hacer para mejorarla.

El Instituto de Economía Internacional es una institución privada sin fines de lucro dedicada al estudio y discusión de la política económica internacional. Su propósito es analizar los problemas importantes en esa área, y desarrollar y comunicar nuevos enfoques prácticos para lidiar con ellos. El Instituto es completamente no partidario.

El Instituto es financiado en gran parte por fundaciones filantrópicas. Las contribuciones institucionales más importantes que se reciben actualmente provienen de la Fundación William M. Keck Jr. y de la Fundación Starr. Varias otras fundaciones y corporaciones privadas contribuyen a los recursos financieros altamente diversificados del Instituto. En nuestro último año fiscal, aproximadamente el $31 \%$ de los recursos del Instituto fue provisto por contribuyentes fuera de los Estados Unidos, incluyendo cerca de un 18\% del Japón. La fundación Charles Stewart Mott proveyó su generoso aporte para este estudio.

La Junta Directiva tiene responsabilidad total por el Instituto y da los lineamientos generales a su programa de investigación, incluyendo la identificación de temas que probablemente serán importantes en el mediano plazo (de uno a tres años) y que deberían ser abordados por el Instituto. El director, trabajando estrechamente con el personal y el Comité Consultivo externo, es responsable por el desarrollo de proyectos particulares y toma la decisión final de publicar un estudio particular.

El Instituto espera que sus estudios y otras actividades contribuyan a construir un fundamento más sólido para la política económica internacional alrededor del mundo. Invitamos a los lectores de estas publicaciones a que nos hagan saber cómo creen que podemos lograr mejor este objetivo.

\section{Fred Bergsten}

Director

Marzo de 2003 


\section{INSTITUTO DE ECONOMÍA INTERNACIONAL}

1750 Massachusetts Avenue, NW, Washington DC, 20036-1903

(202) 328-9000 FAX: (202) 659-3225

C. Fred Bergsten, director

Junta Directiva

* Peter G. Peterson, presidente

* Anthonv M. Solomon. presidente, Comiré Ejecutiov

Leszek Balcerowicz

Conrad Black

Chen Yuan

George David

* Jessica Einhorn Stanley Fischer Maurice R. Greenberg

$\star$ Carla A. Hills

Nobuyuki Idei

Karen Katen

W. M. Keck II

Lee Kuan Yew

William McDonough

William McDonough

Minoru Murofushi

Karl Otto Pöhl

* Joseph E. Robert, Jr.

David Rockefeller

David M. Rubenstein

Renato Ruggiero

* Stephan Schmidheiny

Edward W. Scott, Jr.

George Soros

Lawrence H. Summers

Peter D. Sutherland

Jean Claude Trichet

Laura D'Andrea Tyson

Paul A. Volcker

* Dennis Weatherstone

Edward E. Whitacre, Jr.

Marina v.N. Whitman

Ernesto Zedillo

Ex officio

$\star$ C. Fred Bergsten

Nancy Birdsall

Richard N. Cooper

Diredores henonarios

Allan Greenspan

Reginald $\mathrm{H}$. Jones

Frank E. Loy

George P Shultz
Comité Consultivo

Richard N. Cooper, presidente

Isher Judge Ahluwalia

Robert Baldwin

Barry P. Bosworth

Susan M. Collins

Wendy Dobson

Juergen B. Donges

Barry Eichengreen

Jeffrey A. Frankel

Daniel Gros

Jacob A. Frenkel

Stephan Haggard

David D. Hale

Dale E. Hathaway

Takatoshi Ito

John Jackson

Peter B. Kenen

Anne O. Krueger

Roger M. Kubarych

Paul R. Krugman

Jessica T. Mathews

Rachel McCulloch

Sylvia Ostry

Tommaso Padoa-Schioppa

Dani Rodrik

Kenneth Rogoff

Jeffrey D. Sachs

Nicholes Stern

Joseph E. Stiglitz

Alan Wm. Wolff 


\section{Agradecimientos}

Este estudio se originó durante una conferencia en la Universidad de Princeton en el otoño de 1999, cuando Pedro Pablo Kuczynski expresó a John Williamson su insatisfacción acerca del estancamiento económico en América Latina y le sugirió que era el momento propicio para una nueva evaluación de los problemas, similar a aquella en la que había participado anteriormente (véase Balassa et al., 1986). Fred Bergsten estuvo de acuerdo y el Instituto de Economía Internacional comenzó a trabajar, reuniendo a un equipo de autores conocedores de lo que nosotros consideramos son los principales problemas económicos de la región. El estudio ha tomado más tiempo del previsto debido a otros compromisos, tales como la designación de Kuczynski como ministro de Economía y Finanzas de Perú y el nombramiento de Williamson como director de proyecto para el Informe Zedillo en el 2001.

El grupo sostuvo tres reuniones: dos en Washington (2001 y 2002) y una en Montevideo (2001). Los 15 miembros que han escrito para el estudio, asistieron por lo menos a una de estas reuniones y varios asistieron a las tres. Estas reuniones incluyeron debates intensos e intercambio de ideas y pensamientos entre los autores, por lo que existen muchas deudas intelectuales entre algunos miembros del equipo. Ellos son aquí reconocidos en forma colectiva al inicio de cada capítulo.

Varios otros economistas expertos asistieron a algunas de estas reuniones, en muchos casos para participar en las discusiones de los borradores de los ensayos. Agradecemos las valiosas contribuciones de: C. Fred Bergsten, Jorge Buendia, William R. Cline, Jonathan Eaton, Kimberly Ann Elliot, Richard Feinberg, Carol Graham, Gary Hufbauer, Miguel Kiguel, José Luis Machinea, Antonio Ortiz Mena, Guillermo Perry, Jeffrey Puryear, Pedro Ravela, Paulo Renato Souza, Sebastián Saiegh, Vito Tanzi, Mariano Tommasi, Edwin M. Truman y Roberto Zahler.

También queremos agradecer las valiosas sugerencias de tres colaboradores que no insistieron en el anonimato: William R. Cline, Ricardo Ffrench-Davis y Nora Lustig. 


\section{Visión General:}

\section{Una Agenda para Relanzar el Crecimiento y las Reformas}

John Williamson*

Este puede no ser el peor de los tiempos, pero pocos lo ven como el mejor de los tiempos para América Latina. La región ha vivido otra década de crecimiento lento. Las crisis parecen haberse vuelto cada vez más frecuentes, y las consecuencias de la crisis argentina han sido particularmente dolorosas. La pobreza se redujo en la primera mitad de los años noventa, pero ha empezado a aumentar nuevamente desde 1997. El crecimiento del empleo en el sector formal ha sido agonizantemente lento; la inversión permanece sustancialmente más baja de lo que era en los setenta. La economía mundial está en recesión; los precios de muchos productos primarios han alcanzado recientemente récords de niveles bajos; y los mercados emergentes están fuera de moda para los inversionistas. En muchos países, existe desilusión en relación con los líderes políticos, aunque en la mayoría de casos — según el Latinobarómetro- no con la democracia.

Desde luego, la visión puede estar siendo exageradamente pesimista. El crecimiento revivió en América Latina en la primera mitad de los noventa, hasta que las crisis empezaron a estallar; la inflación, la gran enemiga de los pobres, ha sido conquistada; el crecimiento sigue a las recesiones; los precios de los productos primarios, como los mercados de valores, no bajan para siempre. Las estadísticas nos dicen que el progreso social - medido específicamente en función de longevidad, alfabetismo y mortalidad infantil- continuó aun durante la crisis de la deuda y los noventa (ver tabla 0.1 ).

\footnotetext{
* John Williamson es profesor asociado sentior del Instituto de Economía Internacional desde 1981.
} 
Para poder revisar todo el contenido de esta edición, visite nuestra tienda virtual.
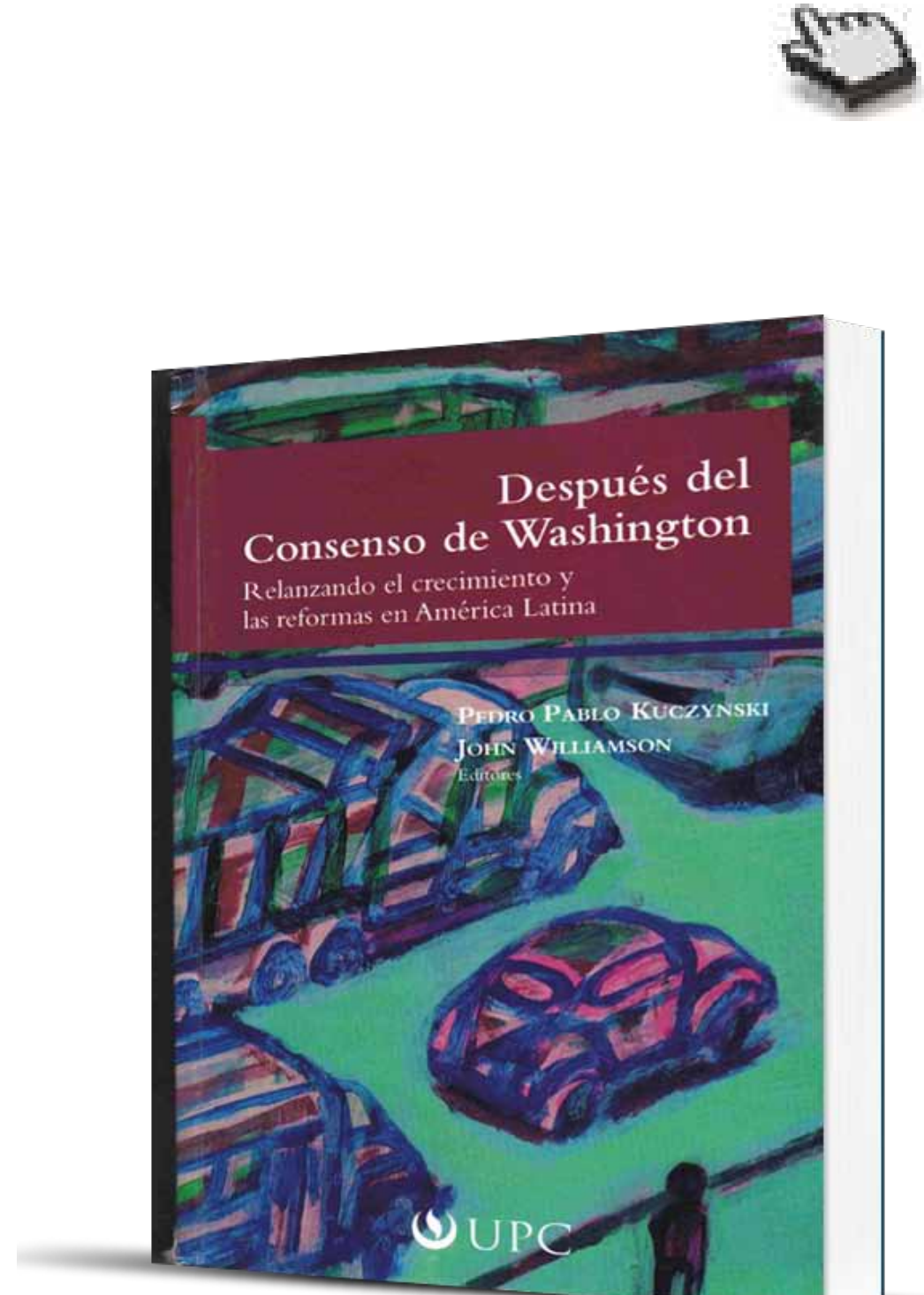